\title{
Variety of the Wave Change in Compound Muscle Action Potential in an Animal Model
}

\author{
Zenya Ito ${ }^{1}$, Shiro Imagama ${ }^{1}$, Kei Ando ${ }^{1}$, Akio Muramoto ${ }^{1}$, Kazuyoshi Kobayashi ${ }^{1}$, \\ Tetsuro Hida ${ }^{1}$, Kenyu Ito ${ }^{1}$, Yoshimoto Ishikawa ${ }^{1}$, Mikito Tsushima ${ }^{1}$, Akiyuki Matsumoto ${ }^{1}$, \\ Satoshi Tanaka ${ }^{1}$, Masayoshi Morozumi ${ }^{1}$, Yukihiro Matsuyama ${ }^{2}$, Naoki Ishiguro ${ }^{1}$ \\ ${ }^{1}$ Department of Orthopaedic Surgery, Nagoya University School of Medicine, Nagoya, Japan \\ ${ }^{2}$ Department of Orthopaedic Surgery, Hamamatsu University School of Medicine, Nagoya, Japan
}

Study Design: Animal study.

Purpose: To review the present warning point criteria of the compound muscle action potential (CMAP) and investigate new criteria for spinal surgery safety using an animal model.

Overview of Literature: Little is known about correlation palesis and amplitude of spinal cord monitoring.

Methods: After laminectomy of the tenth thoracic spinal lamina, 2-140 $\mathrm{g}$ force was delivered to the spinal cord with a tension gage to create a bilateral contusion injury. The study morphology change of the CMAP wave and locomotor scale were evaluated for one month. Results: Four different types of wave morphology changes were observed: no change, amplitude decrease only, morphology change only, and amplitude and morphology change. Amplitude and morphology changed simultaneously and significantly as the injury force increased $(p<0.05)$ Locomotor scale in the amplitude and morphology group worsened more than the other groups.

Conclusions: Amplitude and morphology change of the CMAP wave exists and could be the key of the alarm point in CMAP.

Keywords: Compound muscle action potential; Morphology change; Amplitude

\section{Introduction}

Presently, non-compound muscle action potential specific criteria, such as somatosensory evoked potential (SSEP) criteria (an amplitude decrease $\geq 50 \%$ and $\geq 10 \%$ latency), are often used as the criteria for the warning point of compound muscle action potential (CMAP) $[1,2]$. Several studies have addressed the definition of alarms, with no consensus reached. We have reviewed conventional CMAP alarm points and classified CMAP waveform changes into four grades as novel criteria. Grade 0 is defined as a normal waveform, grade 1 as an amplitude de- crease $\geq 50 \%$ and $\geq 10 \%$ latency, grade 2 as multi-phasing of waveform, and grade 3 as loss of amplitude [3]. The waveform changes from grade 1 to grade 3 in proportion to the severity of injury. The following reports our basic review of the waveform changes using Sprague-Dawley rats.

\section{Materials and Methods}

\section{Modeling of spinal injuries}

Forty-one 8-week-old Sprague-Dawley rats (200-230 g)

Received Mar 9, 2015; Revised Apr 24, 2015; Accepted Apr 24, 2015

Corresponding author: Shiro Imagama

Department of Orthopaedic Surgery, Nagoya University School of Medicine,

65 Tsurumai Showa-ward, Aichi 466-8550, Japan

Tel: +81-52-833-6021, Fax: +81-52-833-6021, E-mail: imagama@med.nagoya-u.ac.jp 
were used. After anesthesia from ether inhalation and intraperitoneal administration of ketamine $(100 \mathrm{mg} / \mathrm{kg})$ and xylazine $(10 \mathrm{mg} / \mathrm{kg}), \mathrm{T} 10$ of the spine of each rat was laminectomized to expose the dura mater, which was pressed with a tension gage (Fig. 1). To create single crush and sustained crush models, stepwise compressions were performed at intensities of 2-140 g for 1-150 seconds. The bladders of the rats were manually voided twice a day for a week after the injury. To prevent infection, $1.0 \mathrm{~mL}$ of Bactramin (Roche, Basel, Switzerland) was mixed in 500 $\mathrm{mL}$ of bottled water provided for hydration for 2 weeks following spinal cord injury. Food was provided on the cage floor, and the rats had no difficulty reaching their water bottles. All animals were treated and cared for in accordance with the Nagoya University School of Medicine Guidelines pertaining to the treatment of experimental animals.

\section{Measurement of control waveform}

The cranial bones were exposed and bores were drilled 3 $\mathrm{mm}$ lateral and $2 \mathrm{~mm}$ posterior to the bregma where bipolar stimulating needles were inserted. A Nihon Koden Neuropack 8 (Nihon Koden Corp., Tokyo, Japan) was used as the stimulator. Except for the stimulus intensity, the stimulus conditions were approximately the same as actual spinal surgery (a train of four pulses at 2-ms intervals, stimulus intensities of 10-60 mA, and 20 additions with a phase switch after 10 additions). A Nihon Koden Neuropack (MEB-2200, ver. 04.02) used in spinal surgery was used to derive the control waveform by inserting

Stimulation: $3 \mathrm{~mm}$ lateral and $2 \mathrm{~mm}$ posterior to the bregma.

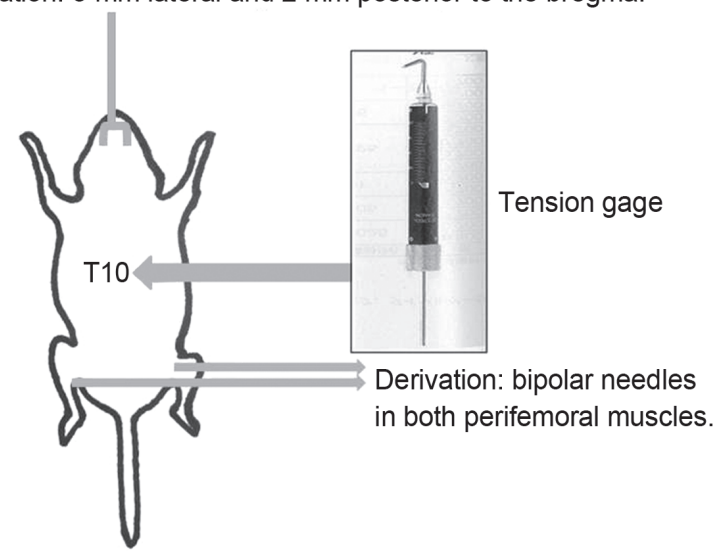

Fig. 1. Spinal cord injury and protocol of compound muscle action potential. bipolar needles in both perifemoral muscles (Fig. 1). The ground electrode was placed subcutaneously between the coil and the recording electrodes.

\section{Derivation and review of CMAP waveform}

Derivation was started immediately after the infliction of injury at every 30 seconds for up to 15 minutes under the same conditions as the foregoing waveform measurement. We reviewed amplitude decrease and morphology change. Morphology change was having occurred in the event of any of the following: change in the number of waves in the waveform, prolonged duration, and shift in the location of the peak latency. For convenience, integrated intensity was defined as compression time multiplied by compression intensity.

\section{Evaluation of the motor function of the lower limbs}

The locomotor performance of 16 animals was analyzed using the Basso, Beattie and Bresnahan (BBB, 0-21 pts) open-field score for 4 weeks ( 1 day, 3 days, 5 days, 1 week, 2 weeks, 3 weeks, and 4 weeks) [4]. The evaluations were made by two blind observers for all analyzed rats.

\section{Statistical analyses}

Statistical analyses were performed with an unpaired twotailed Student's $t$-test for single comparisons and one-way analysis of variance (ANOVA) for multiple comparisons. For the locomotor performance scores, repeated measures ANOVA and the Mann-Whitney $U$-test were used. In all statistical analyses, values of $p<0.05$ were considered to indicate significance. To obtain the data for statistical analyses, the investigators were blinded to the genotypes in all procedures.

\section{Results}

\section{Types of waveform change}

Four different types of waveforms were obtained: no change, amplitude decrease only, morphology change only, and amplitude and morphology change. In the 50 limbs of 25 animals, type 1 accounted for 14\% (7/50 limbs), type 2 for 36\% (18/50 limbs), type 3 for $18 \%$ (9/50 limbs), and type 4 for 32\% (16/50 limbs) (Fig. 2). No 


\section{No change}

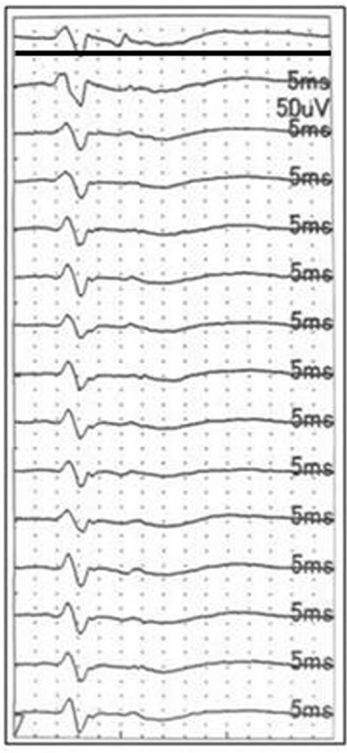

$7 / 50 \operatorname{limbs}(14 \%)$
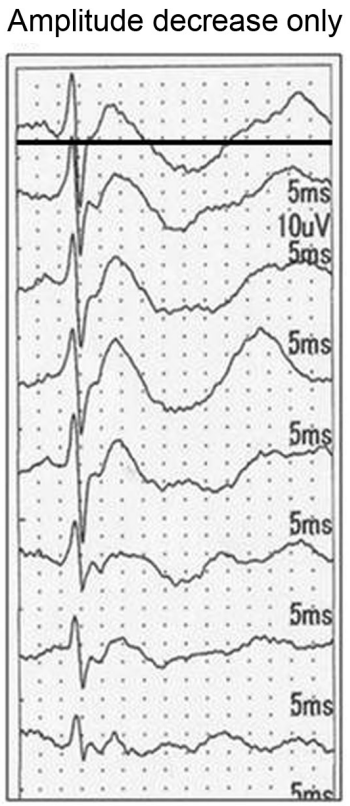

$18 / 50 \operatorname{limbs}(36 \%)$

Fig. 2. The results of wave type.

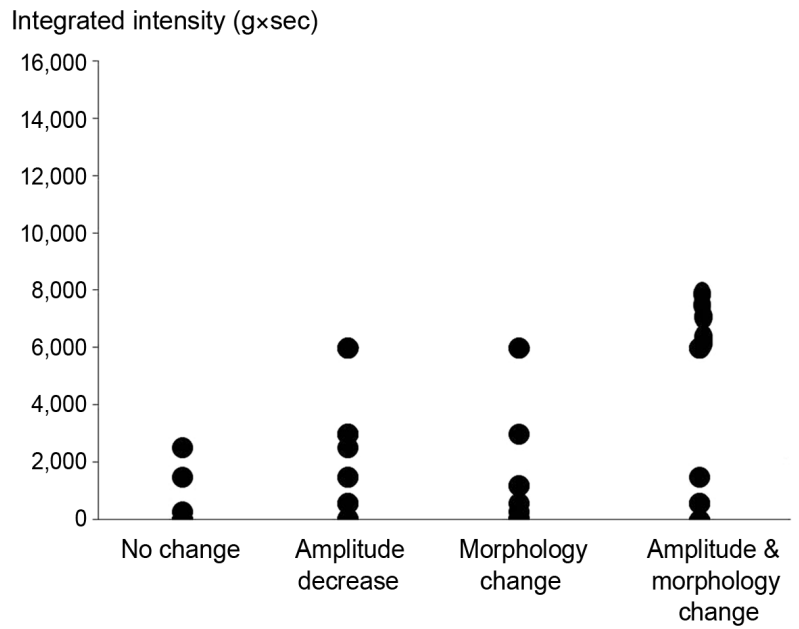

Fig. 3. Relationship between the wave type and the injury force.

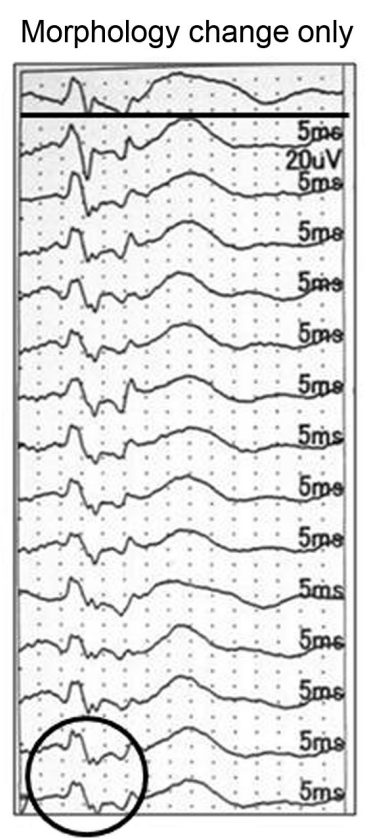

$9 / 50 \operatorname{limbs}(18 \%)$
Amplitude \& morphology change

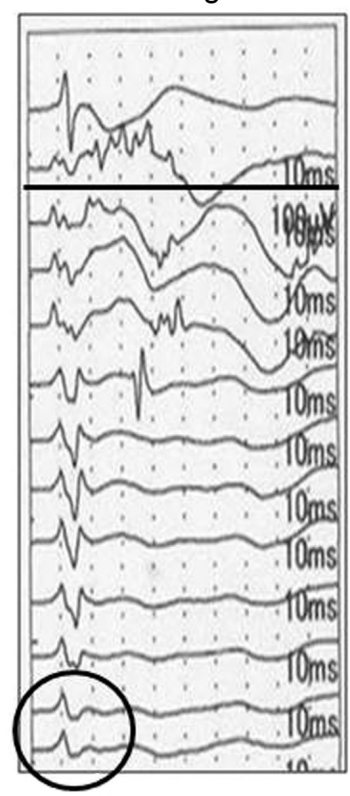

$16 / 50 \operatorname{limbs}(32 \%)$

amplitude decrease and morphology change (Fig. 3). In a box plot, the amplitude group and the morphology group were significantly correlated with high intensities $(p<0.05)$ (Fig. 4).

1) Evaluation of lower limb motor function

At 4 weeks after spinal cord injury, the best result was found in the no change group according to the $\mathrm{BBB}$ score (20). While the amplitude and morphology group (BBB, 13.5) exhibited the strongest degree of paralysis due to its highest integral intensity, there was no significant difference between that group and the amplitude group (BBB, $16.1)$ or the morphology group $(\mathrm{BBB}, 16.5)$ using repeated measure ANOVA $(p=0.07)$. However, the amplitude and morphology group exhibited a significantly higher degree of paralysis up to the third day (Fig. 5).

\section{Discussion}

The CMAP alarm point for stopping surgery remains equivocal [5-8]. Luk et al. [2] applied the same criteria as those for SSEP (amplitude decrease $\geq 50 \%$ and $\geq 10 \%$ latency), Langeloo et al. [9] defined an amplitude decrease $\geq 50 \%$ or more in any muscle as an alarm point, and Sala et al. $[10,11]$ defined waveform loss as an alarm point. Quinones-Hinojosa et al. [12] discussed morphologi- 


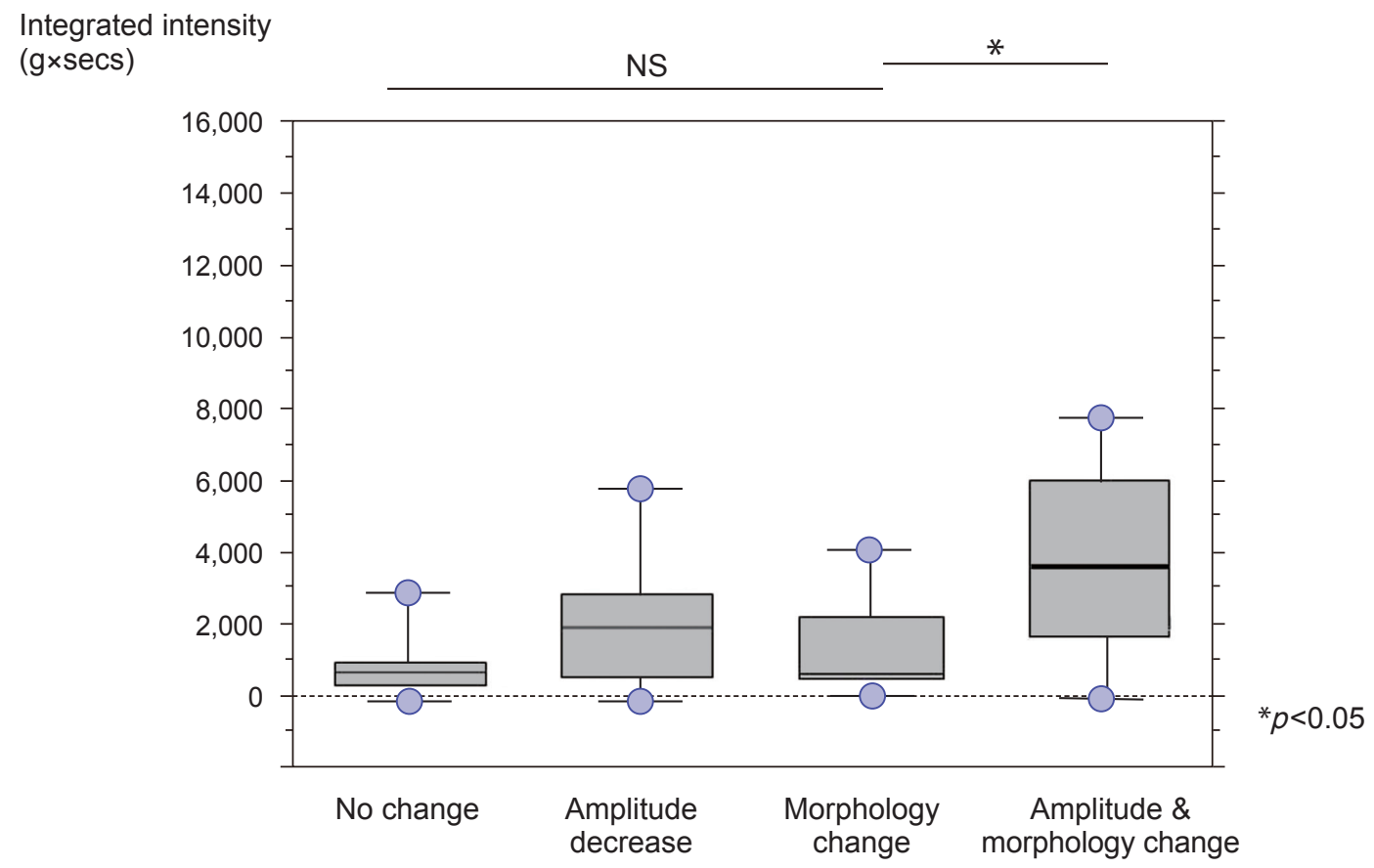

Fig. 4. Relationship between the wave type and the injury force in a box plot. NS, not significant.

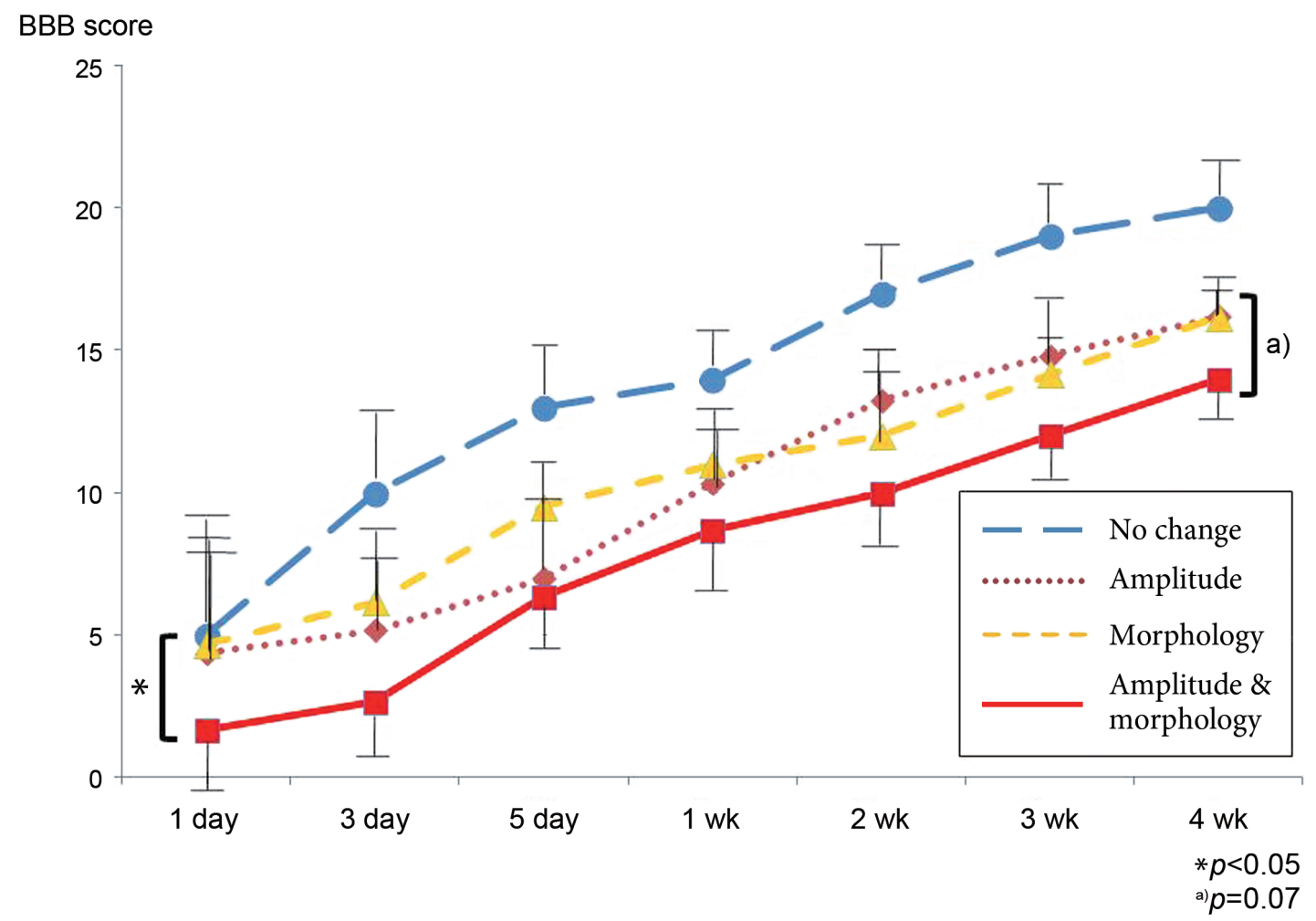

Fig. 5. Relationship between the wave type and Basso, Beattie and Bresnahan (BBB) score.

cal change, defining waveform change from a biphase to a monophase as an alarm point. We proposed defining morphology change as an alarm point [3]. Some authors also reported on a basic study of electrophysiology. How- 
ever, the data only concerned the stimulation method, SSEP alarm point, and electromyography potential [1315]. So, it is clinically unclear and difficult to demonstrate what degree of injury and paralysis of the spinal cord causes morphology change or amplitude decrease. In this study, we used an animal testing model to demonstrate the foregoing.

We were able to classify the waveform into four types. Eighteen percent of the animals exhibited morphology change only without amplitude change. These waveforms were caused by approximately the same force causing the amplitude decrease. This is a novel observation and we believe morphology change should be considered when discussing CMAP alarm points. Additionally, relatively severe injuries tend to be accompanied by amplitude decrease and also morphology change, indicating more critical conditions. Presently, particularly severe paralysis was evident immediately after the injury in the amplitude and morphology groups. This indicates that the concurrence of morphology change and amplitude decrease should be interpreted as a higher level of alarm.

A limitation of this study is the difficulty to explain the mechanism of each waveform. More electrophysiological experiments are needed before clinical applications can be contemplated.

\section{Conclusions}

Change in the morphology and amplitude was accompanied by significant aggravation of paralysis immediately after the injury. It is suggested that morphology change can potentially be one of the alarm points.

\section{Conflict of Interest}

No potential conflict of interest relevant to this article was reported.

\section{References}

1. Bartley K, Woodforth IJ, Stephen JP, Burke D. Corticospinal volleys and compound muscle action potentials produced by repetitive transcranial stimulation during spinal surgery. Clin Neurophysiol 2002;113:7890.

2. Luk KD, Hu Y, Wong YW, Cheung KM. Evaluation of various evoked potential techniques for spinal cord monitoring during scoliosis surgery. Spine (Phila $\mathrm{Pa}$ 1976) 2001;26:1772-7.

3. Ito Z, Imagama S, Sakai Y, et al. A new criterion for the alarm point for compound muscle action potentials. J Neurosurg Spine 2012;17:348-56.

4. Basso DM, Beattie MS, Bresnahan JC. A sensitive and reliable locomotor rating scale for open field testing in rats. J Neurotrauma 1995;12:1-21.

5. Kim DH, Zaremski J, Kwon B, et al. Risk factors for false positive transcranial motor evoked potential monitoring alerts during surgical treatment of cervical myelopathy. Spine (Phila Pa 1976) 2007;32:30416.

6. Lang EW, Beutler AS, Chesnut RM, et al. Myogenic motor-evoked potential monitoring using partial neuromuscular blockade in surgery of the spine. Spine (Phila Pa 1976) 1996;21:1676-86.

7. Gunnarsson T, Krassioukov AV, Sarjeant R, Fehlings MG. Real-time continuous intraoperative electromyographic and somatosensory evoked potential recordings in spinal surgery: correlation of clinical and electrophysiologic findings in a prospective, consecutive series of 213 cases. Spine (Phila Pa 1976) 2004;29:677-84.

8. Weinzierl MR, Reinacher P, Gilsbach JM, Rohde V. Combined motor and somatosensory evoked potentials for intraoperative monitoring: intra- and postoperative data in a series of 69 operations. Neurosurg Rev 2007;30:109-16.

9. Langeloo DD, Lelivelt A, Louis Journee H, Slappendel R, de Kleuver M. Transcranial electrical motorevoked potential monitoring during surgery for spinal deformity: a study of 145 patients. Spine (Phila Pa 1976) 2003;28:1043-50.

10. Sala F, Palandri G, Basso E, et al. Motor evoked potential monitoring improves outcome after surgery for intramedullary spinal cord tumors: a historical control study. Neurosurgery 2006;58:1129-43.

11. Sala F, Bricolo A, Faccioli F, Lanteri P, Gerosa M. Surgery for intramedullary spinal cord tumors: the role of intraoperative (neurophysiological) monitoring. Eur Spine J 2007;16 Suppl 2:S130-9.

12. Quinones-Hinojosa A, Lyon R, Zada G, et al. Changes in transcranial motor evoked potentials during intramedullary spinal cord tumor resection correlate with postoperative motor function. Neurosurgery 2005;56:982-93. 
13. de Blas G, Burgos J, Regidor I, et al. Recording diffusion responses from contralateral intercostal muscles after stimulus-triggered electromyography: refining a tool for the assessment of thoracic pedicle screw placement in an experimental porcine model. Spine (Phila Pa 1976) 2009;34:E391-6.

14. Iyer S, Maybhate A, Presacco A, All AH. Multi-limb acquisition of motor evoked potentials and its ap- plication in spinal cord injury. J Neurosci Methods 2010;193:210-6.

15. Morris SH, El-Hawary R, Howard JJ, Rasmusson DD. Validity of somatosensory evoked potentials as early indicators of neural compromise in rat model of spinal cord compression. Clin Neurophysiol 2013; 124:1031-6. 